REVISTA DE LA ESCUELA DE CIENCIAS DE LA EDUCACIÓN, AÑo 16, NRO. 15, VOL. 1, ENERO A JUNIO DE 2020. PÁGINAS 53 - 62. ISSN $2362-3349$ (EN LÍNEA). EL ESPACIO, EL TIEMPO Y LOS OTROS: EXPERIENCIAS DE FORMACIÓN EN EL PLAN FINES 2 EN LA PROVINCIA DE BUENOS AIRES. MARIA EUGENIA MIGUEZ.

\title{
EL ESPACIO, EL TIEMPO Y LOS OTROS: EXPERIENCIAS DE FORMACIÓN EN EL PLAN FINES 2 EN LA PROVINCIA DE BUENOS AIRES
}

\author{
María Eugenia Míguez* \\ Universidad de Buenos Aires-CONICET, Argentina \\ mariumiguez@gmail.com
}

Recibido: 29/07/2019 Aceptado: 5/11/2019

\section{Resumen}

El siguiente trabajo se desprende de los resultados de mi tesis de Maestría en Educación titulada "Experiencias de formación: sujetos, narrativas y territorios. Un estudio en caso: el Plan FinEs 2 en la Provincia de Buenos Aires". En ella busqué describir, comprender e interpretar las experiencias formativas de cuatro estudiantes del Plan de Finalización de Estudios Secundarios, Fines 2, en los distritos de Vicente López y La Matanza, a través del enfoque (auto) biográfico narrativo en educación.

El propósito de este artículo será reflexionar en torno a las experiencias formativas de los/as estudiantes concibiendo como posibles dimensiones el espacio, el tiempo y los otros. Estos núcleos de sentido que construí a partir del análisis de los relatos de experiencia de estudiantes, buscan visibilizar y valorar aquello que remarcan como formativo de su participación en el plan.

Reconstruir y comprender las experiencias de los/as estudiantes a partir de estas dimensiones puede constituir un aporte para el análisis de esta política educativa en particular, como así también para otras que tengan como objetivo la terminalidad de estudios secundarios para jóvenes y adultos/as. La investigación tomó como referencia temporal la creación del plan FinEs 2 hasta el año 2015.

Palabras clave: Terminalidad educativa - Experiencias de formación - Espacio - Tiempo - Otros.

\section{Abstract}

This paper is part of my master's thesis in education, titled "Experiences of formation: subjects, narratives and territories. A study of cases: the Plan Fines 2 in Buenos Aires province". In the last one I looked for describing, undestanding and reading into the four students' experiences of the Plan of

* Magíster en Educación, Pedagogías Críticas y Problemáticas Socioeducativas por la Facultad de Filosofía y Letras de la Universidad de Buenos Aires. Obtuvo una Beca de Maestría UBA a través del Proyecto de Investigación UBACyT (2014-2017) titulado: La conformación del campo pedagógico: sentidos y disputas en torno de las desigualdades y diferencias en educación. Dirigido por el Dr. Daniel H. Suárez. Sede en el Instituto de Investigaciones en Ciencias de la Educación (IICE- FFyL-UBA). Actualmente ha obtenido una beca de Doctorado CONICET con la dirección de Dra. Laura Pautassi y Co dirección Dra. Esther Levy con sede en la Facultad de Derecho (UBA). Es investigadora adscripta del Instituto "Ambrosio J. Gioja". Facultad de Derecho. UBA. Su formación de grado es Licenciada y Profesora en Ciencias de la Educación (UBA). Actualmente es ayudante de primera en la asignatura "Residencia". Carrera Ciencias de la Educación, FFyL. UBA, y Profesora en Institutos de Formación Docente en la Provincia de Buenos Aires. Más información: https://uba.academia.edu/MaríaEugeniaMíguez 
REVISTA DE LA ESCUELA DE CIENCIAS DE LA EdUCACIÓN, AÑo 16, NRO. 15, VOL. 1, ENERO A JUNIO DE 2020. PÁGINAS 53 - 62. ISSN $2362-3349$ (EN LÍNEA). EL ESPACIO, EL TIEMPO Y LOS OTROS: EXPERIENCIAS DE FORMACIÓN EN EL PLAN FINES 2 EN LA PROVINCIA DE BUENOS AIRES. MARIA EUGENIA MIGUEZ.

Completion Secondary Studies, Fines 2, in Vicente López and La Matanza districts, through a biographic narrative approach in education.

The aim of this article will be to reflect on "space", "time" and "the others" as possible dimensions in the formative experiences of the students. These dimensions search for making visible and valuable all that the students consider formative in the mentioned plan.

To rebuild and understand the students 'experiences through these dimensions could be a contribution to analyze this particular educational policy, also other policies of completion secondary studies for young people and adults. The researching took as temporary reference the establishment of Plan FinEs 2 until 2015.

Keywords: Completion Secondary School - Formative Experiences - Space - Time - Others.

\section{EI Plan de Finalización de Estudios Secundarios "Fines 2"}

El objetivo de este apartado es lanzar las coordenadas, realizar una descripción del Plan FinEs como política pública buscando su contextualización desde la dimensión normativa y territorial para pensar cómo es vivida por los/as estudiantes. Las lecturas vinculadas a cómo se rige e implementa el plan en términos normativos nos permiten reflexionar acerca de los discursos en el campo pedagógico e incluso los efectos que estos generan en las acciones de los sujetos, no está demás remarcar que las disposiciones no conforman solamente un límite, sino también un campo de acción, tensión y negociación, es decir, de agencia para los sujetos.

En Argentina se ha generado una suerte de puja por la inclusión, más visible en momentos de extrema tensión, como en las crisis, en especial la de fines del 2001, pero también presentes en el marco de recuperación económica, como la que caracteriza los periodos posteriores. "Esta puja se encuentra atravesada por nuevas demandas... con el reclamo en término de restitución de derechos o en todo caso, peticiones para que el contenido del derecho se efectivice" (Pautassi, 2012, p. 278).

En la última década el Estado asume un rol principal en la educación argentina. Esto lo observamos a partir de una mayor presencia de lo social en la legislación con mayor apertura al reconocimiento de derechos (Vassiliades y Saforcada, 2011). La afirmación de la educación como derecho social fue impulsada en términos legislativos por la sanción de la Ley de Educación Nacional (LEN) 26.206 en 2006 y, para el caso bonaerense, de la Ley de Educación Provincial (LEP) 13.688 en 2007. En ellas aparece el Estado como principal garante del derecho a la educación. Este asume un papel principal en asegurar el acceso a todos los ciudadanos y ciudadanas a la información y al conocimiento como instrumentos centrales de la participación, en un proceso de desarrollo, con crecimiento económico y justicia social (Ley 26.206, 2006). Para el caso bonaerense el Estado asume un papel principal en la provisión y garantía de una "educación inclusiva y de calidad" para todos los habitantes de la provincia (Ley 13.688, 2007, art. 5). En consonancia, con dichas leyes se consagra la obligatoriedad de la educación secundaria (Cap. IV, art. 29). El Ministerio de Educación, Ciencia y Tecnología y las autoridades jurisdiccionales competentes asegurarán el cumplimiento de la obligatoriedad escolar a través de alternativas institucionales, pedagógicas y de promoción de derechos, que se ajusten a los requerimientos locales y comunitarios, urbanos y rurales, mediante acciones que permitan alcanzar resultados de calidad equivalente en todo el país y en todas las situaciones sociales (Ley 26.206, 2006, art. 16).

En este marco de obligatoriedad de la educación secundaria, el Estado toma como prioritaria la terminalidad educativa. En 2008 se pone en marcha en el territorio nacional, el Plan de Finalización de Estudios Primarios y Secundarios "FinEs". En la primera etapa conocida como FinEs 1 (año 2008), sus acciones estuvieron destinadas a dar respuesta a jóvenes y adultos/as que cursaron el último año de la educación secundaria como alumnos/as regulares y adeudaban materias sin haber alcanzado el título. La segunda etapa conocida como FinEs 2 (2009-2011), a jóvenes y adultos/as que no iniciaron o no completaron su educación primaria y/o secundaria (Lozano y Kurlat, 2014; Finnegan y Brunetto, 2014; Finnegan y Brunetto, 2015). De acuerdo con ello, se impulsa, en la Provincia de Buenos Aires, el Plan Provincial de Finalización de Estudios Obligatorios y Vuelta a la Escuela, y dentro de este Plan Provincial, el Plan FinEs 2 comienza a tener mayor protagonismo.

Ahora bien, ¿qué particularidades adquiere este plan de terminalidad educativa en el territorio? ${ }^{1}$. Tal como menciona el artículo 47 de la LEN las acciones de los programas educativos de la Modalidad de Jóvenes y Adultos se llevarán a cabo en articulación con distintos ministerios. En este caso, fue necesario el trabajo intersectorial entre el Ministerio de Educación de la Nación, la Dirección General de Cultura y

\footnotetext{
${ }^{1}$ En mi tesis de Maestría en Pedagogías Críticas y Problemáticas Socioeducativas (FFyL, UBA) he desarrollado la categoría territorio(s) como campo de acción pedagógica, esto quiere decir que las experiencias formativas se arraigan en territorios. Estos no son neutrales ni armónicos, tampoco las experiencias que crecen allí. No son solo espacios geográficos que podemos delimitar nítidamente en un mapa. Estos territorios generan en los sujetos pertenencia, identidad. En este sentido, el territorio es un espacio vivido, habitado por lo tanto histórico, cultural y pedagógico.
} 
REVISTA DE LA ESCUELA DE CIENCIAS DE LA EdUCACIÓN, AÑO 16, NRO. 15, VOL. 1, ENERO A JUNIO DE 2020. PÁGINAS 53 - 62. ISSN $2362-3349$ (EN LÍNEA). EL ESPACIO, EL TIEMPO Y LOS OTROS: EXPERIENCIAS DE FORMACIÓN EN EL PLAN FINES 2 EN LA PROVINCIA DE BUENOS AIRES. MARIA EUGENIA MIGUEZ.

Educación (DGCyE) de la Provincia de Buenos Aires y el Ministerio de Desarrollo Social (la Coordinación de Formación para el Programa Ingreso Social con Trabajo) de la Nación.

En 2010, a través de la Resolución Provincial 3520/10, se establece que esta propuesta educativa estará destinada a jóvenes y adultos/as mayores de 18 años que no cumplimentaron la educación secundaria, en especial a las cooperativas que forman parte del programa de Ingreso Social con Trabajo "Argentina Trabaja" ${ }^{2}$, con el objetivo de ofrecer a los/as trabajadores/as organizados/as la posibilidad de cursar y finalizar la Educación Secundaria (Art.2). Tal como plantea Finnegan y Brunetto (2014),

Los inicios del Plan en la Provincia se ligan a la voluntad política de dar respuesta a la gran proporción detectada de cooperativistas de este Programa, con los niveles educativos obligatorios incompletos, que justificó que -inicialmente- estuviera restringido a estos sujetos, aunque -tambiéndirigido a integrantes de entidades gremiales (Finnegan y Brunetto, 2014, p. 6).

En la misma resolución (3520/10) y en otras (Res. 444/12) aparecerá la figura de sedes educativas, estos serán los espacios donde podrá desarrollarse el plan, tales como: instituciones educativas de nivel primario o secundario de la modalidad de adultos o espacios públicos o privados que cumplan las condiciones necesarias para el desarrollo de las clases, supervisado por el/la Inspector/a de la modalidad.

Originalmente, en la Provincia de Buenos Aires el plan funcionaba en el marco de articulaciones entre el Estado y la sociedad civil (Levy, 2012). Estas articulaciones lograban que el Plan FinEs llegase a las personas de barrios populares, entre ellos los/as titulares del programa de "Argentina Trabaja". Aparecían allí actores sociales llamados "referentes", que pertenecían a movimientos sociales y/o políticos, religiosos, ONGs, sindicatos y organismos del sector público como el Ministerio de Desarrollo Social, y que eran quienes realizaban la inscripción y el seguimiento de personas jóvenes y adultas dentro del plan. Entre otras tareas, los/as referentes armaban los listados y solicitaban la apertura de una sede o comisión a la Inspección de Adultos de su jurisdicción. La comisión de alumnos/as era la unidad más pequeña del plan, se insertaba en el marco de una sede, en la que podía haber una o más comisiones. Las organizaciones sociales proveían este espacio físico y su costo de mantenimiento. Estas sedes se constituían como espacios en movimiento (Míguez, 2018), conectados con otros espacios barriales. Fue así que el Plan FinEs funcionó en escuelas, sociedades de fomento, clubes, merenderos, iglesias, fábricas, polos productivos y otros lugares de trabajo.

La propuesta de FinEs 2 (secundaria) en la Provincia de Buenos Aires comenzó con 3 años de cursada organizados cuatrimestralmente; el plan de estudios fue el aprobado por la Resolución $N^{\circ}$ 6321/95 con orientación en Ciencias Sociales y Gestión y Administración. Cada CENS (Centro de Educación de Nivel Secundario $)^{4}$ referente sería el responsable de emitir las certificaciones correspondientes. Los/as docentes tutores serían designados por acto público con la presentación de un proyecto pedagógico de la asignatura a dictar y su retribución mensual sería la de un Profesor de Secundaria. A partir de la Disposición 99/12, se reconoce el trayecto educativo ya recorrido y aprobado de los/as jóvenes, adultos/as y se configuraba una tabla de equivalencias. Esto implicó que ingresaran estudiantes en distintos años al plan, de acuerdo a las equivalencias aprobadas. Por su parte, la Resolución 444/12 señalaba que "la modalidad es de carácter presencial y los alumnos asistirán dos días por semana".

Luego, en el año 2013 se incorporan como parte del Argentina Trabaja las mujeres destinatarias del Programa "ELLAS HACEN" (dependiente del Ministerio de Desarrollo Social). Este programa estuvo destinado a 100 mil mujeres, para que pudieran formar parte de una cooperativa de trabajo, además, como requisito obligatorio, las mujeres titulares debían completar la primaria o la secundaria. El convenio realizado allí fue entre el Ministerio de Desarrollo Social de la Nación y la DGCyE a través de sus jefaturas regionales y distritales para que las titulares pudieran finalizar sus estudios en el Plan Fines 2.

A partir de esta síntesis podemos pensar que el Plan Fines se manifiesta como política flexible en términos de adecuarse a las necesidades de los sujetos. A través de la normativa notamos que ha incluido al menos discursivamente, a distintos sujetos, primero "deudores de materias", luego a personas que no habían finalizado la primaria y/o la secundaria, y a sujetos pertenecientes a distintos programas sociales. Es decir, a todos aquellos que la escuela "ha dejado afuera". Dichos sujetos poseen características heterogéneas, por edades, género, trayectorias. Esta heterogeneidad se traduce en diversidad de experiencias respecto a la participación de los/as estudiantes en el Plan FinEs. Consideramos que conocer las

\footnotetext{
${ }^{2}$ En la última década en Argentina, se impulsó una línea de política social destinada a sectores débilmente integrados al mercado formal de trabajo que retomaba -discursivamente- el ideario de la economía social y que estaba dirigida a promover la inclusión social con trabajo de población vulnerada, a través de acciones emprendidas desde el Ministerio de Desarrollo Social de la Nación. En este marco, en 2009 se lanza el Programa Ingreso Social con Trabajo "Argentina Trabaja" (PRIST-AT) que tendrá por objetivo la promoción del desarrollo económico y la inclusión social (Res. 3182/09).

${ }^{3}$ Los Centros Educativos de Nivel Secundario (CENS) datan de 1973 a partir de la creación de la rama técnica específica, designada como "Dirección de Educación de Adultos" - por Decreto № 4626/73 y dependiente de la Subsecretaría de Educación. Están destinados a personas que quieran retomar o iniciar los estudios secundarios y cuentan con un plan de estudios de tres años de duración.
} 
REVISTA DE LA ESCUELA DE CIENCIAS DE LA EdUCACIÓN, AÑo 16, NRO. 15, VOL. 1, ENERO A JUNIO DE 2020. PÁGINAS 53 - 62. ISSN $2362-3349$ (EN LÍNEA). EL ESPACIO, EL TIEMPO Y LOS OTROS: EXPERIENCIAS DE FORMACIÓN EN EL PLAN FINES 2 EN LA PROVINCIA DE BUENOS AIRES. MARIA EUGENIA MIGUEZ.

experiencias de formación en el plan desde las voces de sus protagonistas puede ser un aporte para repensar esta política y desde allí la escuela.

La primera resolución aprobada a inicios de la gestión "Cambiemos" en el Ministerio de Educación y Deportes, referente al Plan FinEs 2, fue la N 178/16 que sostiene dar continuidad al "Plan de Finalización de Estudios Primarios y Secundarios _FinEs_-" para el periodo 2016-2019. Para el caso de la Provincia de Buenos Aires, la continuidad la otorga la Resolución 713/17. Dicha normativa marca tres modalidades: FinEs Trayecto Primaria, FinEs Trayecto Secundario y FinEs Deudores de materias. Además, dispone una carga horaria mínima de 18 horas cátedra, es decir, tres días por semana para la línea Trayecto Secundario (esto quiere decir que se agrega un día más de cursada a la propuesta original) y un mínimo de 15 alumnos/as para la apertura de comisiones. Esta apertura será analizada de acuerdo con la matrícula existente en las reuniones de Unidades Educativas de Gestión Distrital y serán aprobadas posteriormente por la Dirección de Adultos de la DGCyE. A su vez, las inscripciones se realizarán únicamente en las Bocas Únicas Distritales (BUD) o a través de la web (Adultos 3.0). También se dicta la Resolución 114/16 que deja sin efecto la 99/12, vinculada al sistema de equivalencias, y la Resolución 115/16, que establece el marco reglamentario para el acceso y nombramiento de docentes tutores del Plan FinEs.

Nos preguntamos cómo impactan los cambios en el plan llevados adelante a partir del cambio de gestión en 2015 en las experiencias de los/as estudiantes, entre ellos el giro hacia la virtualidad (Adultos 3.0), la falta de referencia territorial (las inscripciones se realizan únicamente a través de la Boca Única Distrital indicada por el/la inspector/a de la modalidad), la cursada de tres veces por semana en espacios escolares (se redujeron significativamente las sedes emplazadas en espacios barriales), la fusión de comisiones (sólo funcionan comisiones con un mínimo de 15 estudiantes). Es en ello en lo que me encuentro trabajando actualmente y será objeto de futuros análisis.

\section{Investigar la experiencia desde un abordaje (auto) biográfico narrativo}

La pregunta por la experiencia nos conduce hacia los sentidos que construyen los sujetos sobre su vida, sobre su estar en el mundo. La pregunta por la experiencia nos interpela e invita a reflexionar sobre aquello que nos pasa (Larrosa, 2009). Es que eso que nos pasa deja huellas, marcas en nuestro cuerpo, genera sentimientos, emociones, que son difíciles de borrar. Las experiencias guardadas en nuestra memoria aparecen en nuestras acciones, nos alertan, nos enseñan e indican determinados caminos. Ellas portan un saber. Nos transportan al lugar de lo conocido, de la seguridad. O por el contrario, nos recuerdan aquel lugar al que no queremos volver. Ahora bien, ¿qué implica tornar visibles las experiencias de los sujetos en el campo pedagógico?

Tal como señala Larrosa (2009) la cuestión de la experiencia tiene muchas posibilidades en el campo educativo, éste se constituye como un territorio surcado por relaciones de poder y de saber y luchas por la hegemonía (Suárez, 2011). En el caso de la investigación que informa este trabajo la pregunta por las experiencias se vincula a la posibilidad de explorar, visibilizar y valorar las voces de los/as estudiantes del Plan FinEs 2, como así también comprender los sentidos que construyen a partir de sus experiencias. De este modo, trabajar con las experiencias de los/as estudiantes del Plan Fines 2 posee un triple propósito. En primer lugar, abonar a la posibilidad y la necesidad de incorporar estas voces como legítimas en el campo de saber y poder pedagógicos. En este sentido, además de cuestionar las formas convencionales de pensar y hacer en materia de investigación educativa, las versiones más críticas de las perspectivas interpretativas aportaron ideas y estrategias valiosas para establecer una conversación más vivificante y horizontal con los actores de las prácticas educativas y, sobre todo, para afrontar el desafío de desarrollar modalidades más democráticas de producción de conocimientos pedagógicos. En segundo lugar, esta particular forma de reconstruir la experiencia permite, a su vez, reconstruir el territorio educativo a partir de los relatos de experiencia y las historias de vida de los mismos sujetos que lo transitaron y habitaron. Conociendo y comprendiendo las experiencias de formación, podremos aportar claves para re- pensar el espacio educativo que se indaga. Por último, que el actor y narrador de su propia historia pueda, al reflexionarla y escribirla, ordenarla, integrarla y comprenderla de otro modo. Esta posibilidad de re-pensar y re-escribir la historia, constituye uno de los elementos centrales de la investigación con perspectiva narrativa y (auto) biográfica, que muchas veces resulta también un dispositivo de formación. En términos de Delory Momberger (2009), el "actor biográfico" se torna educador de sí mismo.

Ahora bien, ¿qué implica investigar la experiencia teniendo en cuenta los propósitos mencionados? ¿Cómo investigar la experiencia? Scott (2001) advierte acerca de que:

No son los individuos los que tienen la experiencia, sino los sujetos los que son constituidos por medio de la experiencia. En esta definición la experiencia se convierte entonces no en el origen de nuestra explicación, no en la evidencia definitiva (porque ha sido vista o sentida) que fundamenta lo conocido, sino más bien en aquello que buscamos explicar, aquello acerca de lo cual se produce el conocimiento. Pensar de esta manera en la experiencia es darle historicidad, así como dar historicidad a las identidades que produce. (Scott, 2001, p. 49) 
REVISTA DE LA ESCUELA DE CIENCIAS DE LA EdUCACIÓN, AÑo 16, NRO. 15, VOL. 1, ENERO A JUNIO DE 2020. PÁGINAS 53 - 62. ISSN $2362-3349$ (EN LÍNEA). EL ESPACIO, EL TIEMPO Y LOS OTROS: EXPERIENCIAS DE FORMACIÓN EN EL PLAN FINES 2 EN LA PROVINCIA DE BUENOS AIRES. MARIA EUGENIA MIGUEZ.

Lo que señala Scott (2001) nos permite problematizar la experiencia como categoría para no caer en una mirada ingenua, idealizada y descontextualizada de las vivencias de los sujetos. En este sentido, Arnaus (2008) nos alerta sobre:

Tomar conciencia del peligro de que, a través de mi trabajo pueda mantener o reproducir la jerarquización teoría-práctica. Mantener o reproducir la diferencia entre quienes analizan la práctica y quienes la practican y en consecuencia legitimar la dependencia intelectual (y por tanto la división social del trabajo) de unas personas sobre otras. (Scott, 2001, p. 73)

Entonces me pregunto, ¿Podemos reconstruir experiencias sin reconstruir las nuestras como investigadores? ¿Es posible reconstruir experiencias sin reconocer al otro como sujeto de saber?

Es que investigar la experiencia implica necesariamente la reflexión sobre la propia experiencia de investigación. Esto no intenta ser un juego de palabras. Al contrario, lo que intentamos resaltar es el compromiso y la implicancia a la que nos lleva indagar sobre aquellas cosas que para el otro tienen sentido, sobre lo que el otro recuerda y lo que decide olvidar, sus silencios, emociones. Resulta difícil investigar la experiencia si no estamos dispuestos a ser atravesados por ella. En relación a ello Arnaus (2008) rescata del libro "El antropólogo como autor" la advertencia que señala Geertz (1989) en relación con la posición objetiva, distanciada y de privilegio de los autores de las etnografías provocaba acusaciones de insensibilidad, de tratar a la gente como objetos, de escuchar las palabras pero no la música". Ahora bien, ¿cómo debemos actuar, que acciones debemos desarrollar si, como investigadores, pretendemos escuchar las palabras para comprender la música? Considero que esto supone un desafío, intentar no traicionar la confianza y la intimidad que compartimos para realizar la indagación. No cristalizar ni imponer al relato de los protagonistas categorías extranjeras, sino más bien presentar una realidad abierta al diálogo, que suscite reflexiones pedagógicas, que arroje pistas para comprender aquellas experiencias educativas. Esto significa el respeto y el aprecio mutuos entre los/as participantes y la construcción de relaciones horizontales de sujeto a sujeto. Las entrevistas narrativas ${ }^{3}$ me permitieron crear estos espacios de intimidad, de confianza mutua. Además, resultó positivo para el proceso de investigación mi participación en el plan, como docente primero y coordinadora después. Esto me permitió generar una red de contactos, estudiantes, profesores/as, referentes, inspectores/as, algunos/as de ellos/as se convirtieron en informantes claves para la investigación.

Fue a partir de los viajes por el territorio, las reuniones con docentes, inspectores, las observaciones en sedes de FinEs, las entrevistas con estudiantes, que conocí a los/as cuatro casos que elegí: Mary, Lucas, Marcelo y Gaby. Cada perfil aportaba una mirada distinta al plan. Mary y Gaby eran referentes de sede en cada uno de sus barrios, con la particularidad de que Gaby pertenecía además a un programa social. Lucas era egresado del plan y se había convertido en profesor de matemáticas allí. Marcelo vivía en Vicente López temporalmente, pero era oriundo de La Matanza y cursaba el plan allí. A partir de un episodio complicado que involucraba a su sobrino había abandonado su casa, su barrio para vivir en Vicente López. Sin embargo, había algo que no podía dejar, sus compañeros/as del FinEs, por ellos/as volvía a La Ferrere dos veces por semana para estudiar.

Construí un guión de preguntas comunes para ellos/as. Luego del primer encuentro desgrababa la entrevista y armaba nuevas preguntas para cada uno/a. Utilizaba las desgrabaciones para elaborar la primera versión del relato de experiencia. En los siguientes encuentros compartía con ellos/as ese relato mientras narraban vivencias que complementaban esa primera versión. Los relatos nos unían, y, a la vez, nos permitían tomar distancia. Poner en papel aquellas palabras que circulaban en nuestros encuentros habilitaba un espacio común, el de la narrativa. Allí, en ese territorio podíamos negociar los sentidos, leer y releer para opinar, para emocionarnos, para discutir y generar "campos de inteligibilidad" (Bolívar, 2002).

El trabajo hermenéutico, de reinterpretación y análisis de los relatos de experiencias de formación, construidos a partir del material empírico, me permitió construir núcleos de sentido vinculados al "tiempo, el espacio y los otros". Estos núcleos constituyen lo que llamaré dimensiones de las experiencias de formación. Por un lado, concebimos la formación en sentido amplio, como un proceso individual y fundamentalmente social que se desarrolla en un tiempo y espacio. Las experiencias no son lineales ni tampoco suponen un punto de partida y de llegada. Lo que podemos sostener a partir de aquí es que los espacios, tiempos y el vínculo con los otros han habilitado en los/as estudiantes del Plan FinEs 2 -en Mary, Lucas, Marcelo y Gabyciertas experiencias formativas.

\footnotetext{
${ }^{4}$ Para Connelly y Clandinin (2008) la entrevista narrativa es la clave para la autocomprensión y desde esta, para el cambio. Señalan que estas entrevistas son interactivas y que los investigadores tratan de apresar temas generadores en la vida del informante: los hilos de la trama que dan sentido a los numerosos cambios que acompañan su vida. Desde el punto de vista de la experiencia, el proceso se parece más a un grupo de apoyo mutuo para poner en vigencia un cambio en la vida propia.
} 
REVISTA DE LA ESCUELA DE CIENCIAS DE LA EdUCACIÓN, AÑo 16, NRO. 15, VOL. 1, ENERO A JUNIO DE 2020. PÁGINAS 53 - 62. ISSN $2362-3349$ (EN LÍNEA). EL ESPACIO, EL TIEMPO Y LOS OTROS: EXPERIENCIAS DE FORMACIÓN EN EL PLAN FINES 2 EN LA PROVINCIA DE BUENOS AIRES. MARIA EUGENIA MIGUEZ.

\section{Los/as estudiantes: miradas compartidas}

Es difícil no pensar en las recurrencias. Con esto quiero decir que a medida que iba desarrollando las entrevistas con los/as estudiantes era inevitable compararlas ya que encontraba en sus relatos hilos comunes. Si bien no es la intención de este trabajo establecer categorías para ubicar cada vida narrada, sí constituye un elemento interesante poder pensar en que estas vidas tienen puntos de encuentro. Cabe aclarar que las experiencias no son lineales, ni armoniosas. Las experiencias formativas son zigzagueantes, interrumpidas, dañadas. Cada una nos permite comprender lo que pasa en el interior del proceso de formación, captando datos y con ello sentidos sobre fenómenos que serían inaccesibles por otros medios. Cada historia fue trabajada en vista de liberar los momentos bisagras (Jossó, 2014) de sus vidas, especialmente de su formación. Estos puntos de inflexión (Kornblit, 2007) son momentos vitales identificados por el sujeto o investigador/a a partir de los cuales el itinerario biográfico de la persona toma un rumbo distinto o inicia una nueva etapa. Tal como sostiene Kornblit (2007) no solo debemos identificar estos cambios, sino más bien comprender por qué los sujetos han llegado a realizar esos cambios en determinados momentos de sus vidas.

Entonces, lo que tienen en común estas historias es que los cuatro estudiantes forman o formaron parte de un plan de terminalidad educativa, el FinEs 2. En este sentido, los cuatro vieron sus trayectorias educativas interrumpidas en algún momento de sus vidas. Claro que, en cada caso, las razones son diferentes como así también los recorridos que han realizado en la escuela. En las cuatro narrativas aparece un sabor amargo, un sentimiento de tristeza respecto de haber tenido que abandonar sus estudios. Los cuatro sintieron frustración por tener que dejar o no empezar la escuela secundaria. Esto se traducirá como una asignatura pendiente: "quise, pero no pude". Ese no poder está conformado en cada relato por distintas circunstancias de vida, sin embargo, la que prima es "era estudiar o trabajar". Los trabajos que pudieron conseguir en su juventud se vincularon al trabajo informal, pesado: ayudante de cocina, vendedor ambulante, empujando un carro o descargando rollos de telas para una fábrica textil. Las edades en que cada uno comenzó a trabajar también son similares, luego de terminar la primaria, entre los 10 y 12 años. Los intentos por finalizar sus estudios secundarios se desarrollaron en propuestas para adultos tales como CENS y semipresencialidad, en otro caso a través del programa $\mathrm{COA}^{5}$. En cada historia el acercamiento a estas propuestas es diverso. Lo común podría estar dado en que en sus relatos expresan las reiteradas veces que lo intentaron. Respecto al vínculo de sus familias de origen con la escuela, en los cuatro relatos advertimos que sus madres y/o padres no han finalizado sus estudios. En el caso de Mary, su madre era analfabeta, al igual que la de Gaby. Los padres de Marcelo y Lucas finalizaron solamente la primaria. Esto no impide que su percepción sobre ellos sea la de padres y madres que portan saberes legítimos y valiosos.

Existen miradas compartidas acerca de ciertas características del Plan. Por un lado, las cuatro historias resaltan aspectos sobre el espacio y tiempo que propone el Plan FinEs 2 para finalizar la secundaria. Por otro lado, aparece como positivo el respeto y puesta en valor de los saberes previos de los/as estudiantes, el debate y el aprendizaje colectivo que a partir de allí proponen los/as profesores/as. El vínculo con otros que se produce a través del contacto, el encuentro, la conformación de grupos, funciona como contención y motor para alcanzar el objetivo de finalizar los estudios.

\section{Sentidos en torno al espacio, el tiempo y los otros. Romper las fronteras de lo escolar}

De acuerdo con el marco normativo señalado el Plan FinEs 2 Secundaria deberá desarrollar sus sedes educativas en todos los distritos de la provincia de Buenos Aires, estas sedes estarán emplazadas en escuelas de educación primaria, secundarias, organismos del estado, organizaciones gremiales, empresariales, universidades, ONG, agrupaciones varias. De acuerdo a ello se abre en el Centro Educativo Complementario (CEC) "La Escuelita", una sede del Plan FinEs 2 en el barrio "El Ceibo" de Vicente López, allí cursó Mary, estudiante y referente de esa sede. Mary piensa en los vecinos del barrio que estarían interesados en esta propuesta. Fue así que llegó a la inspectora de Adultos de Vicente López. "Éramos como 23 para terminar el secundario", cuenta. Así, comenzó a tejer redes en el distrito y se reunió con la inspectora para plantearle la necesidad de "bajar" el FinEs al barrio. También se transforma en sede del plan la escuela de educación popular "Yo sí puedo" en el barrio La Juanita -Gregorio de Laferrere- donde terminó la secundaria Lucas. Luego, él dictaría clases de Matemática en un Club cercano a su hogar, también convertido en sede de FinEs. En el caso de Marcelo cursó sus estudios secundarios en una iglesia evangélica en Gregorio de Laferrere. El Plan se desarrollaba allí ya que la iglesia prestaba el espacio físico. Gaby, estudiante y titular del programa Argentina Trabaja cursó FinEs en el Obrador 24 de Laferrere y fue referente de una comisión que funcionó en la sede "Fidel Castro".

Los espacios son vividos por los sujetos de distintas maneras, en el caso de Mary y Gaby su relación con la sede es especial. Ambas son referentes y el cuidado hacia el espacio es notorio. Están encargadas de

\footnotetext{
${ }^{5}$ Los Centros de Orientación y Apoyo se crean en el año 2003 (Resolución DGCyE № 3039/03) como un programa específico para promover la finalización y acreditación del nivel secundario. Estos Centros funcionan en diversas escuelas de la Provincia de Buenos Aires brindando acompañamiento pedagógico a aquellas personas jóvenes y adultas que quisieran aprobar las asignaturas pendientes para acreditar un año o nivel.
} 
REVISTA DE LA ESCUELA DE CIENCIAS DE LA EdUCACIÓN, AÑO 16, NRO. 15, VOL. 1, ENERO A JUNIO DE 2020. PÁGINAS 53 - 62. ISSN $2362-3349$ (EN LÍNEA). EL ESPACIO, EL TIEMPO Y LOS OTROS: EXPERIENCIAS DE FORMACIÓN EN EL PLAN FINES 2 EN LA PROVINCIA DE BUENOS AIRES. MARIA EUGENIA MIGUEZ.

abrir y cerrar la sede. Siempre remarcan a los/as estudiantes la importancia de mantener el espacio en condiciones para la cursada. En el caso de Marcelo, la sede también guarda un vínculo especial con él. Su pareja, Mónica, practica la religión evangelista. Al escuchar la radio cristiana advierten un anuncio sobre el Plan FinEs como propuesta para terminar los estudios secundarios. No les llamó la atención que el FinEs se desarrollara en una iglesia, esto más bien guardaba cierta relación con el anuncio. Los/as estudiantes refieren a estos espacios como escuela. Si bien las características edilicias difieren bastante de las de una institución escolar, son reiteradas las veces que en las entrevistas dicen: "vamos a la escuela o al colegio". El FinEs 2 se forma como un espacio en movimiento, conectado con otros espacios, fundamentalmente, organizaciones sociales: iglesias, ONG, sindicatos, merenderos, clubes, por nombrar algunos. Estos espacios influyen en la vida de los sujetos y en su formación. Al habitarlos, Mary, Lucas, Marcelo y Gaby, no solo se apropian, sino que también los transforman. Estos espacios, entre otras cosas, posibilitaron la finalización de sus estudios secundarios.

Foucault (1966) se refería a las heterotopías como espacios otros, es decir, lugares reales, pero fuera de todos los lugares. Se conoce este término a raíz de conferencias radiofónicas (una se llamó El cuerpo utópico y la otra heterotopías), ambas datan de 1966. "Las heterotopías son espacios otros, diferentes, que les permiten a los sujetos no solo contraponerse a los órdenes de los espacios establecidos, sino también arreglárselas con el mundo" (Runge Peña, 2015, p. 137). El hincapié está puesto en que el espacio no es neutro y blanco, sino por el contrario, es vivido y vivenciado. Podríamos pensar las heterotopías como espacios de resistencia. Este espacio social juega un papel en la formación del sujeto, en su despliegue como ser corporal, temporal y espacial. Tal como señala Runge Peña (2015) el espacio es parte fundamental del proceso de construcción de la identidad y de la formación del individuo. Así, al referirnos al espacio, estamos en un ámbito en el que se conjugan vivencias, percepciones, configuraciones, sentidos, construcciones y relatos.

A su vez, los/as estudiantes conciben de diversas maneras el tiempo. El tiempo no está asociado únicamente con la frecuencia en la que cursan. También se vincula, tomando el marco normativo, con los años que deben cursar de acuerdo al sistema de equivalencias. Tal como aparece en los relatos Mary y Marcelo debieron cursar tres años para finalizar. En el caso de Lucas y Gaby, solo un año. Esta posibilidad se produce a partir de la ya mencionada Disposición 99/12. Si bien la normativa establece un tiempo determinado, los sujetos viven ese tiempo y las posibilidades que les ofrece de diversas maneras. De esta forma podríamos hablar de un tiempo "objetivo" determinado por los límites que establece el plan en sus normativas (duración del plan en términos de años, cantidad de cuatrimestres, etcétera) y un tiempo "subjetivo o vivido" por los sujetos. Podemos comprender cómo se articulan ambos tiempos a través del tiempo narrado. Este tiempo no es lineal y unívoco. El tiempo en las biografías también es interpretado y necesita de interpretación y reflexión. Pocas veces somos dueños de nuestro tiempo. En los relatos aparece cómo es vivido por los sujetos. Aquí algunos fragmentos de los relatos que dan cuenta de ello:

Claro, yo tenía que rendir dieciséis materias, tenía mi trabajo, atender a mis hermanos, ¿cómo hacía para estudiar? así que no fui más. (Relato Gaby)

Para nosotros era una asignatura pendiente. Primero los chicos eran chicos y yo siempre prioricé a mis hijos, el tema del colegio y cuando ellos ya eran adolescentes empezamos a ocuparnos de nosotros. (Relato Marcelo)

FinEs fue la solución a muchas familias del barrio, porque había chicos que no terminaron los estudios y FinEs les dio la posibilidad de terminar los estudios fuera del horario de trabajo, quizás la gente trabaja a la mañana o a la tarde, y hoy en día FinEs está de noche, tarde noche, y la gente puede terminar de estudiar. (Relato Gaby)

En los relatos aparece una dimensión más a las ya mencionadas -espacio y tiempo- la dimensión vincular, la relación con el Otro. En este sentido, los relatos dan cuenta de cómo se van conformando aprendizajes cooperativos, grupalidad y el respeto y valor por los saberes previos. Sin embargo, estas construcciones colectivas no estarán exentas de contradicciones. Los vínculos no suelen ser lineales ni determinados de una vez y para siempre, el vínculo, al igual que la experiencia, se encuentra en permanente construcción. El vínculo de los sujetos con el conocimiento, con los saberes previos, vínculos mediados por otros, por docentes, compañeros/as, vecinos/as, familiares que vuelven a encontrarse en estos espacios.

Yo siempre tengo preguntas que dan para el debate. Queremos seguir estudiando. No ser alguien porque ya lo soy, pero si tener herramientas para llegar a otros. De eso se trata la vida de poder ayudarnos entre nosotros. (Relato Mary)

En la escuela como en el barrio existen relaciones de reciprocidad. Estos vínculos se caracterizan, muchas veces, por su solidaridad y confianza. Son pares, pero también son vecinos/as, forman parte de una 
REVISTA DE LA ESCUELA DE CIENCIAS DE LA EdUCACIÓN, AÑO 16, NRO. 15, VOL. 1, ENERO A JUNIO DE 2020. PÁGINAS 53 - 62. ISSN $2362-3349$ (EN LÍNEA). EL ESPACIO, EL TIEMPO Y LOS OTROS: EXPERIENCIAS DE FORMACIÓN EN EL PLAN FINES 2 EN LA PROVINCIA DE BUENOS AIRES. MARIA EUGENIA MIGUEZ.

comunidad. Las relaciones que se dan en la comunidad no son lineales y únicamente amorosas, surgen también los conflictos, los celos, las mezquindades. Así lo señala una docente "los problemas del barrio se trasladan al aula", sin embargo, la convivencia allí era distinta. "Pueden enojarse y discutir, pero con respeto".

Aparece allí, una bandera blanca, un espacio neutral donde poder resolver conflictos familiares, vecinales. Esto por lo menos es lo que resaltó la docente como una estrategia frente a los conflictos que excedían los temas curriculares de la clase, "como se conocen se ayudan mucho, vos tenés que dar una consigna, entregar una tarea y faltó alguien y te dicen: "Bueno yo se la doy, la veo mañana" "llevamos juntos los chicos al colegio". "Creo que eso hace un soporte interesante, sobre todo a la constancia, a que sigan viniendo, esta cosa grupal."

Al respecto Gaby sostiene que:

Antes, cuando entré acá no me gustaba escuchar a nadie, por todo me enojaba, hablaba muy mal, y de repente fuimos conociendo gente, a los profesores. Al ser grandes no nos retaban, nos decían que teníamos que hablar mejor, corregir las formas, leer más y hablar menos. (Relato Gaby)

-Bien, está bueno, ¿y de estas clases que fue lo que más te gustó?

-Trabajar en grupo. Cinco o seis mujeres de treinta años con pibes, haciendo el afiche y te sacas un diez. Es lo mejor que hay ¿cómo hace esta mina para estudiar, con los pibes, con la casa? fue una experiencia buenísima porque nunca pensé que se podía. Como a mí me gusta dibujar, hice todos los afiches. Nosotros teníamos a Marcela en Derecho, ella me preguntaba “¿cómo hiciste?”, porque con seis pibes, tu marido, tu casa y no es que me lo sabía de memoria, fue una lección hermosa. (Relato Gaby)

El grupo cobra en estos espacios vital importancia. Sostiene, genera pertenencia e identidad. El grupo, cuyos integrantes son heterogéneos en edad, sexo, intereses personales, trayectorias, tienen un objetivo en común, terminar la escuela secundaria, y terminarla todos/as juntos/as.

Para mí es como que uno tiene un sentido de pertenencia. Arrancamos ahí con este grupo, y la verdad que nos sentimos contenidos, más por esta situación, donde uno viene pasando este problema, encima buscar un lugar donde tenés que arrancar de nuevo y empezar a conocer nuevos compañeros, amigos, nuevos estudiantes, nuevos profesores. En realidad, los profesores son nuevos constantemente, pero el tema es empezar a buscar nuevamente esos amigos o esas personas que medianamente ya te están conteniendo. Por eso te digo que es como que tengo un sentido de pertenencia, también es porque como que soy parte de ellos, me siento así, me siento una persona de igual a igual. (Relato Marcelo)

Rosa María Torres (2004) sostiene que construir una Comunidad de Aprendizaje implica revisar la distinción convencional entre escuela y comunidad. "La escuela es, por definición, parte de la comunidad, se debe a ella, está en función de ella; docentes y alumnos son al mismo tiempo agentes escolares y agentes comunitarios." (Torres, 2004, p. 3). En esta clave Villa (2019) sostiene que:

El plan FinEs representa la posibilidad de hablar de una nueva politización de lo pedagógico, de nuevas modalidades de gestión de las instituciones y de la enseñanza, de nuevos y diferentes compromisos respecto de la circulación de la palabra, que extienden ese derecho y bien social que es la educación; la posibilidad de encontrar un espacio de desarrollo para una verdadera pedagogía social, territorial... que dé impulso a la construcción de nuevos saberes para las comunidades. (Villa, 2019, p. 29)

Esta definición resulta interesante para comprender los espacios de FinEs en la comunidad y la relación con el otro que allí se construye. En este sentido, reconocer que cada grupo y comunidad tiene recursos, necesidades y realidades específicas, determina proyectos educativos y culturales también específicos, ajustados a cada realidad y contexto. "Antes que 'modelos' a adoptarse acríticamente o a proponerse como respuestas universalmente válidas, se promueve la construcción y experimentación de experiencias diversas, con capacidad para inspirar a otros, más que para ser replicadas" (Torres, 2004, p. 4). De lo que se trata es de vivir una experiencia formativa no solo en términos de conocimiento, sino además de vínculos con el otro. Y siguiendo los relatos podemos comprender que esos vínculos involucran tensiones, conflictos, solidaridad, respeto y diversidad. Vínculos que no expresan solamente competencia e individualidades, vínculos que no tienen que ver con trabajar contra los otros para alcanzar un objetivo, que solo uno o unos pocos podrán conseguir. En este sentido, el mérito se resignifica de tal modo que uno solo no podrá alcanzar el objetivo si todos los demás no lo alcanzan también.

Estos núcleos de sentido no tienen pretensión de generalización, sino, por el contrario, lo que busco es transmitir cómo llegué a las categorías nómades para pensar el Plan FinEs 2 y las experiencias de los/as estudiantes allí. En este sentido, considero que las categorías construidas tendrán fertilidad teórica en la 
REVISTA DE LA ESCUELA DE CIENCIAS DE LA EdUCACIÓN, AÑo 16, NRO. 15, VOL. 1, ENERO A JUNIO DE 2020. PÁGINAS 53 - 62. ISSN $2362-3349$ (EN LÍNEA). EL ESPACIO, EL TIEMPO Y LOS OTROS: EXPERIENCIAS DE FORMACIÓN EN EL PLAN FINES 2 EN LA PROVINCIA DE BUENOS AIRES. MARIA EUGENIA MIGUEZ.

medida en que exista, entre el/la investigador/a y los sujetos protagonistas, una escucha atenta e intercambios mediados por el diálogo. Las fertilidades teóricas se darán, entonces, si son ellos los que toman la palabra. Si logramos contar más que ser contados, si conseguimos narrar nuestras experiencias para reflexionar sobre el qué y por qué de lo que somos. En definitiva, la experiencia es siempre una interpretación y requiere una interpretación. Esta nunca será neutral u objetiva, sino producto de nuestros puntos de vista, de nuestros condicionamientos de vida.

\section{Reflexiones finales}

He pretendido a lo largo de este trabajo reflexionar en torno a las experiencias formativas de los/as estudiantes concibiendo como posibles dimensiones el espacio, el tiempo y los otros. Para ello comencé con la descripción normativa y territorial del plan FinEs 2 Secundaria para conocer y comprender las posibilidades que brinda la normativa y cómo éstas son vividas por los estudiantes. Ellos/as han señalado la posibilidad de "formar parte", de cumplir con una "asignatura pendiente" que era finalizar los estudios secundarios, la posibilidad de escuchar y aprender del otro. El Estado a partir de esta política pública educativa logró, por lo menos en parte, habilitar otras experiencias formativas. Esto no quiere decir que el proceso haya sido positivo en su totalidad, como tampoco que el Plan no sea perfectible. Sin embargo, la apropiación del Plan hace que eso que falta también los/as interpele a movilizarse. Si bien consideramos que muchas de las características que diferencian al Plan FinEs 2 de otras ofertas educativas para adultos, podría deberse a que se constituye como un programa flexible. Esto resulta una apuesta riesgosa, dado

que no debiera depender solo de la coyuntura política lo que resulta una deficiencia de la modalidad en cuanto a garantizar el derecho a la educación de los/as jóvenes y adultos/as.

El enfoque auto biográfico narrativo en educación en el que se basa la investigación que enmarca este trabajo nos permite repensar los modos de "hacer" investigación, el cómo investigar. En este punto, rompe con la relación sujeto-objeto de la epistemología tradicional en tanto el investigador y los participantes son sujetos, con deseos, miedos, emociones, son sujetos sentipensantes. Esto significa que quien desea conocer la realidad no puede estar en el contexto social "observando" como "objetos de estudio" a los actores sociales. En el proceso de investigación todos los participantes son reconocidos como sujetos de saber.

La apuesta por visibilizar las voces de los estudiantes: Mary, Lucas, Marcelo y Gaby, no con la intención de producir generalizaciones que, una vez enunciadas, permitieran explicar luego cualquier otro caso singular. Tampoco la intención fue presentarlas como casos atípicos o como héroes/heroínas sociales. Más bien lo que intentaron los relatos fue reconstruir la experiencia formativa de cada uno para reflexionar sobre la potencialidad de la producción de relatos polifónicos en el campo pedagógico.

Lo que hemos pretendido en este trabajo es abrir interrogantes, preguntas, relatos que nos inviten a seguir pensando otros modos de "hacer escuela", que nos permitan escuchar lo que tienen para decir los estudiantes sobre su formación. A ponernos en el lugar de estudiantes para poder recibir, investigar y motivarnos a seguir aprendiendo con el otro. También, la invitación a explorar otros modos de decir en investigación, de ahí la importancia de los relatos, la intertextualidad. Esta herramienta nos permite contar experiencias, historias en un lenguaje inclusivo, un lenguaje que nos acerca, intriga e invita a seguir interpretando nuestras vivencias.

\section{Referencias bibliográficas}

Arnaus, R. (2008). Voces que cuentan y voces que interpretan: Reflexiones en torno a la autoría narrativa en una investigación etnográfica. En: Larrosa, J. y otros. Déjame que te cuente. Ensayos sobre narrativa y educación. Barcelona, España: Laertes.

Bolívar, A. (2002). ¿De nobis ipsis silemus? Epistemología de la investigación biográfico narrativa en educación. En: Revista Electrónica de Investigación Educativa [en línea]. Volumen 4 (№1), 1-26. Recuperado de http://redie.uabc.uabc.mx/vol4no1/contenido-bolivar.html.

Brunetto, C.; Finnegan, F.; Kurlat, S. y Lozano, P. (julio 2014). Contribuciones y limitaciones del Plan "FinEs 2 Secundaria. Perspectivas de estudiantes y profesores. En: Revista Novedades Educativas. № 283, pp. 54-58.

Connelly, F. y Clandinin, D. J. (2008). Relatos de experiencia e investigación narrativa. En: Larrosa, J. y otros. Déjame que te cuente. Ensayos sobre narrativa y educación. Barcelona, España: Laertes.

Delory- Momberger (2009). Biografía y educación. Figuras del individuo-proyecto. Buenos Aires: Facultad de Filosofía y Letras, Universidad de Buenos Aires y Consejo Latinoamericano de Ciencias Sociales.

Finnegan, F. y Brunetto, C. (2015). Contexto, gobierno y actores sociales en la producción de la política de Educación de Jóvenes y Adultos: el Plan FinEs Secundaria. En: Prácticas pedagógicas y políticas educativas: investigaciones en el territorio bonaerense. (pp. 379-400) Buenos Aires. Argentina: UNIPE.

Josso, M. C. (2014). Proceso autobiográfico de (trans)formación identitaria y de conocimiento de sí. En: Revista Mexicana de Investigación Educativa. vol. XIX (№ 62), julio-septiembre de 2014. pp.735-761.

Kornblit, A. L. (2007). Metodologías cualitativas en ciencias sociales. Buenos Aires: Editorial Biblos.

Larrosa, J. y Skliar, C. (comps). (2009). Experiencia y alteridad en educación. Rosario, Santa Fe, Argentina: Homo Sapiens.

Levy, E. y Bermudez, A. (2012) De la empleabilidad a la autogestión. La relevancia de la educación formal en el Programa "Argentina Trabaja". En: ¿Más derechos, menos marginaciones? Políticas sociales y bienestar en la Argentina (pp. 181-217). Buenos Aires: Biblos.

Míguez, M. E. (2018). Experiencias de formación: sujetos, narrativas y territorios: Un estudio en caso, el Plan FinEs 2 en la Provincia de Buenos Aires (Tesis de Maestría). Universidad de Buenos Aires, Argentina. 
REVISTA DE LA ESCUELA DE CIENCIAS DE LA EdUCACIÓN, AÑO 16, NRO. 15, VOL. 1, ENERO A JUNIO DE 2020. PÁGINAS 53 - 62. ISSN $2362-3349$ (EN LÍNEA). EL ESPACIO, EL TIEMPO Y LOS OTROS: EXPERIENCIAS DE FORMACIÓN EN EL PLAN FINES 2 EN LA PROVINCIA DE BUENOS AIRES. MARIA EUGENIA MIGUEZ.

Pautassi L. (2012) La exigibilidad de derechos en contextos de marginación social. El lugar de la política pública. En: ¿Más derechos menos marginaciones? Políticas sociales y bienestar en la Argentina (pp. 275-301). Buenos Aires: Biblos.

Runge Peña, A. (2015). Los espacios pre escolares vivenciados y narrados por niños y niñas: Estudios sobre la formación de la infancia desde un enfoque fenomenográfico-narrativo. En: Murillo Arango, G. J. (comp.). Narrativas de experiencia y educación y pedagogía de la memoria (pp.135-171) Buenos Aires, Argentina: Consejo Latinoamericano de Ciencias Sociales.

Scott, J. (2001). Experiencia. En: La Ventana (N¹3). pp.42-73.

Suárez, D. H. (2011). Relatos de experiencia, saber pedagógico y reconstrucción de la memoria escolar. La documentación narrativa de experiencias pedagógicas en la formación docente. En: Alliaud, A. y Suárez, D. H. (comps.). El saber de la experiencia. Narrativa, investigación y formación docente. pp. 93-138. Buenos Aires, Argentina: Facultad de Filosofía y Letras, Universidad de Buenos Aires y Consejo Latinoamericano de Ciencias Sociales.

Torres, M. R. (2004). Comunidad de aprendizaje repensando lo educativo desde el desarrollo local y desde el aprendizaje. En: Simposio Internacional sobre Comunidades de Aprendizaje. Simposio llevado a cabo en Barcelona Forum 2004, Barcelona, España.

Vassiliades, A. y Saforcada, F. (2011). Las leyes de educación en los comienzos del siglo XXI: del neoliberalismo al postconsenso de Washington en América del Sur. En: Educ. Soc. Vol. 32 (Nº 115), (abril-junio, 2011) 287-304. Recuperado de: http://www.cedes.unicamp.br.

Villa, A. (2019). FinEs 2: Una pedagogía social, una pedagogía del territorio, una didáctica integrada. En: García, D (comp.). Didáctica y pedagogía de la Educación Secundaria de jóvenes y adultos. Prácticas de la Educación Popular en el sistema educativo formal. Memorias del FinEs 2 (pp. 27-38). Buenos Aires: Novedades Educativas.

\section{Marco normativo y fuentes}

Ley N²6.206 de Educación Nacional. Congreso Nacional de Argentina, 14 de diciembre de 2006.

Ley № 26.058 de Educación Técnico Profesional. Congreso Nacional de Argentina, 8 de septiembre de 2005.

Ley $\mathrm{N}^{\circ} 26.075$ de Financiamiento Educativo. Congreso Nacional de Argentina, 9 de enero de 2006.

Ley N 13.688 de Educación Provincial. Congreso de la Provincia de Buenos Aires, 27 de junio de 2007.

CFE, Resolución 66/08, Anexo V.

CFE, Resolución 178/16.

Resolución 3520/10. Dirección General de Cultura y Educación (DGCyE), Provincia de Buenos Aires, octubre 2010.

Resolución 6321/95. DGCyE, diciembre 1995.

Resolución 444/12. DGCyE, abril 2012.

Disposición 99/12 DGCyE. agosto 2012.

Disposición 60/13 DGCyE. noviembre 2013.

Resolución 713/17. DGCYE, abril 2017.

Disposición 114/16. DGCyE, noviembre 2016.

Disposición 115/16. DGCyE, noviembre 2016. 